\title{
¿HACIA UN NUEVO PARADIGMA BIBLIOTECARIO? EL NUEVO ORDEN DIGITAL
}

Concha Varela-Orol

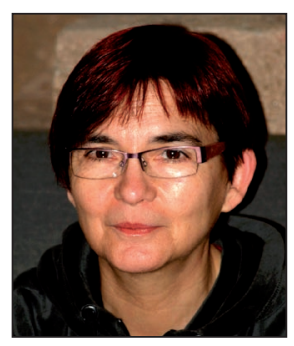

Concha Varela-Orol es doctora en historia y miembro del Cuerpo Facultativo de Archivos y Bibliotecas. Fue directora de la Biblioteca Universitaria de Santiago de Compostela y participó en la formación de la red Rebiun. En la actualidad es profesora de la titulación de Biblioteconomía y Documentación de la Universidad de A Coruña. Sus líneas de investigación son: historia de las bibliotecas y de las ciencias documentales, y gestión de bibliotecas, sobre las que ha publicado diversos libros y artículos.

Universidade da Coruña. Departamento de Humanidades Dr. Cabrera, s/n. 15403 Ferrol (A Coruña) concepcion.varela@udc.es

\section{Resumen}

Teniendo en cuenta los cambios que dieron lugar al paradigma bibliotecario actualmente vigente, se analizan los modelos de bibliotecas emergentes en el marco de la información y comunicación digital, vistos como una adaptación a una nueva sociedad surgida de un uso intensivo de las TIC y al orden económico que la sustenta. Se tienen en cuenta especialmente las nuevas alianzas de las bibliotecas entre ellas, pero también con otros servicios y con instituciones como los archivos y museos.

\section{Palabras clave}

Bibliotecas integradas, Centros de recursos para el aprendizaje y la investigación, CRAI, Convergencia de bibliotecas, Archivos y museos.

\section{Title: Towards a new paradigm in librarianship? The new digital order}

\begin{abstract}
The library models emerging in a framework of digital information and communication technologies are analyzed, taking into account the changes that leaded to the current library paradigm. The models are seen as an adaptation of the libraries to a society based on an intensive use of the ICTs and the economic order that supports it. The article focuses on the new alliances among libraries (joint-use libraries), and also with other services (information commons) and institutions like archives and museums (LAM).
\end{abstract}

\section{Keywords}

Joint-use libraries, Information commons, Libraries, archives and museum convergence, LAM.

Varela-Orol, Concha. "¿Hacia un nuevo paradigma bibliotecario?: El nuevo orden digital”. El profesional de la información, 2011, septiembre-octubre, v. 20, n. 5, pp. 564-570.

\section{Introducción}

Hoy es algo común hablar del fuerte impacto que la tecnología está teniendo en las bibliotecas. Iniciado con la automatización de sus procesos y productos, y continuado por el empleo de las nuevas formas de comunicación -espacios web y las redes sociales para alojar su información o servicios a la comunidad, contenidos digitales adquiridos o licenciados, pero también producido por ellas mismas-, está introduciendo cambios que van más allá de los visibles a primera vista. Hasta tal punto debe reposicionarse en la denominada sociedad de la información y el conocimiento, que se impone un nuevo modelo bibliotecario. En las siguientes páginas intentaremos analizar la situación, aunque desde luego las respuestas actuales están lejos de constituir soluciones definitivas.

Este trabajo intenta preguntarse si caminamos hacia un nuevo paradigma porque en el mundo de las bibliotecas se están produciendo cambios de tal trascendencia que el modelo tradicional que hasta ahora se usaba para entender y gestionar los procesos empieza a mostrar su incapacidad en muchos puntos. Dado que todo paradigma implica una estructura y unas reglas de transformación que hagan posibles 
las dinámicas de gestión, todo parece apuntar a la necesidad de ir elaborando un modelo alternativo con capacidad heurística y de toma de decisiones en este momento. No pretendo elaborar tal modelo ahora, sino únicamente señalar algunos procesos y datos a modo de materiales para su construcción. La referencia de fondo que utilizo no es kuhniana, fundamentalmente estática, sino procesual que articula elementos de distinta procedencia. El que utilice el concepto "paradigma" para referirme a un ámbito restringido como es el de las bibliotecas tiene detrás las "teorías de medio rango" o "rango restringido" de Merton (1968) y el concepto de "campo" de Bourdieu (1994); los procesos de anticipación y sospecha introducidos están inspirados en Foucault (1968). Para pensar la
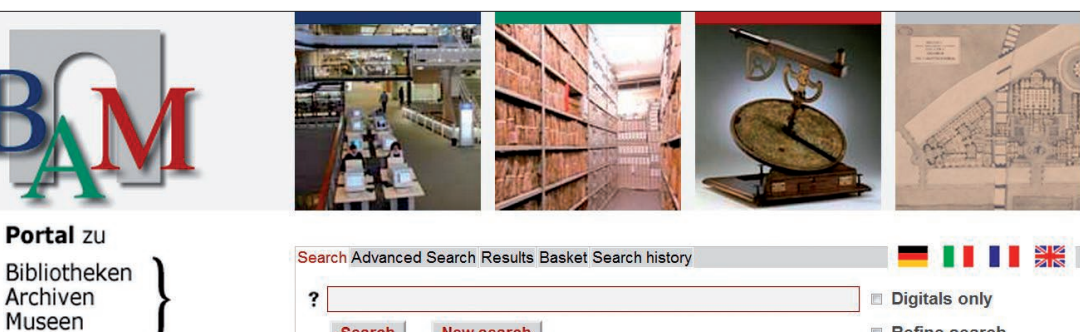
Museen

| start About us FAQ Questionnaire Contact / Participation Disclaimers

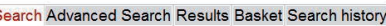
Search New search Digitals only Refine search

The BAM Portal enables the search in the Collections of the participating Libraries, Archives, Museums and Other sources in Germany. Most information in the data is only accessible in the German longuage but it is not necessary to use the German umlaut an special character

\section{Search example}

Neuigkeiten und Hinweise

Fliegende Blätter Kunst- und
Satirezeitschriften de
19 und frühen 20 19. und frühen 20 Jahrhunderts

Schätze des Altonaer Museums Graphische Sammlung

Die Graphische Sammlung der Universitätsbibliothek Heidelberg umfasst mehr als 4.000 Bläter und Aufnahmen. Sie beinhaltet überwiegend Portraits, Ansichten, Karten und Pläne aus einem Zeitraum von fünf Jahrhunderten. Neben Holzschnitten, Kupfer- und Stahlstichen, Radierungen, Lithographien und Zeichnungen enthalt sie auch zahlreiche Fotografien. Ihren Schwerpunkt hat die Graphische Sammlung im Heidelberger und Kurpä̈lzischen Raum. So sind von Heidelberger Professoren in der Portraitsammlung beispielsweise Holzschnitte und Kupferstiche, die bis in die Frühzeit der Universitat zurückreichen, aber auch Lithographien und Fotografien aus dem 19. und frühen 20. Jahrhundert

http://ww.bam-portal.de dialéctica entre paradigma y realidad concreta me inspiro en el modelo marxiano de "modo de producción" y su relación con la "formación económico-social", así como en las remodelaciones posteriores que sobre este tema realizaron Gramsci (1974) y Bastide (1998) que, como es sabido, dedicaron especial atención al análisis de la superestructura ideológica.

Algunos de los cambios que afectan a la identidad de las bibliotecas no son iguales en el transcurso de la historia, sobre todo si analizamos los períodos anteriores a la especialización científica de los siglos XVII y XVIII. Especialmente en el último, las instituciones denominadas por los anglosajones "de la memoria" cambian radicalmente su modelo, en un camino hacia la apertura al público, la autonomía de recursos financieros, el carácter enciclopédico de sus colecciones y la profesionalización de su personal (Varela, 2009). Antes de analizar la situación actual introducimos brevemente algunas notas históricas.

\section{Mirando al futuro desde el pasado}

El origen de las bibliotecas, los museos y los archivos puede rastrearse en las culturas más antiguas, pero tal y como hoy los conocemos, instituciones poseedoras de información puesta al servicio del conocimiento, la educación y el disfrute de la ciudadanía, tienen su origen en el nacimiento de la esfera pública y en las políticas diseñadas en los siglos XVIII y XIX para la puesta en valor y difusión del patrimonio (Chapron, 2009; Varela, 2009). En sus orígenes bibliotecas y archivos nacen fundidos en sus colecciones y funciones, los gabinetes de curiosidades reúnen objetos de todo tipo, y las bibliotecas muestran monetarios, instrumentos científicos, globos terráqueos y mapas, etc., todavía hoy presentes en muchas de ellas. La denominación de museo que reciben a veces las bibliotecas en los siglos XVII y XVIII indica claramente que la diferenciación entre instituciones es tardía.

Su diversificación actual deriva de la especialización característica del siglo XVIII, que basará específicamente en los soportes y formatos informativos y comunicativos la división de las tres instituciones, lo que traerá como consecuencia el desarrollo de tratamientos y prácticas documentales autónomas. Bien es cierto que la existencia de distintos soportes que ha sustentado la diferencia entre archivos, bibliotecas y museos, no fue nunca tan radical como la simplista diferencia entre manuscrito/impreso/objeto, sino que siempre hubo territorios comunes (publicaciones oficiales, documentos personales...), pero sin duda fue el soporte empleado de forma predominante el que marcó la dicotomía más fuertemente percibida, que posicionaría por un lado a bibliotecas y archivos, territorios del texto escrito, y museos, territorio del objeto. La frecuencia con que hasta el día de hoy dentro de determinadas organizaciones el mismo equipo humano es responsable de los servicios de archivos y bibliotecas es indicativa de la importancia del soporte informativo y de hasta qué punto pervive el recuerdo de un pasado común. Pero incluso las prácticas realizadas con sus recursos informativos, como es el caso del patrimonio, se comparten entre archivos y bibliotecas y se separan en el campo de los museos. Manteniendo las tres instituciones su función preservadora, los museos se enfocan hoy a interpretar, o proponer interpretaciones, y transmitir los valores de sus piezas a un público cada vez más amplio, como puede verse en las exposiciones temporales o en el papel que juegan en la industria del turismo de masas, mientras bibliotecas y archivos orientan su patrimonio fundamentalmente al apoyo administrativo y a la investigación.

La especialización no se detuvo a lo largo de los siglos XIX y $X X$, y los tipos dentro de cada una de las instituciones de que hablamos no hicieron sino incrementarse, estableciéndose una compartimentalización entre distintas categorías de bibliotecas (escolares, públicas, etc.) que ha llegado hasta hoy mismo, y cuya base está en la dependencia institucional y la segmentación de grupos de usuarios cuyas demandas informativas y culturales se presumen distintas.

De la triple categorización que establecía la Encyclopédie francesa entre bibliotecas particulares, de comunidades (monasterios, colegios universitarios, etc.) y públicas -correspondiendo éstas al nuevo modelo nacido con la emer- 


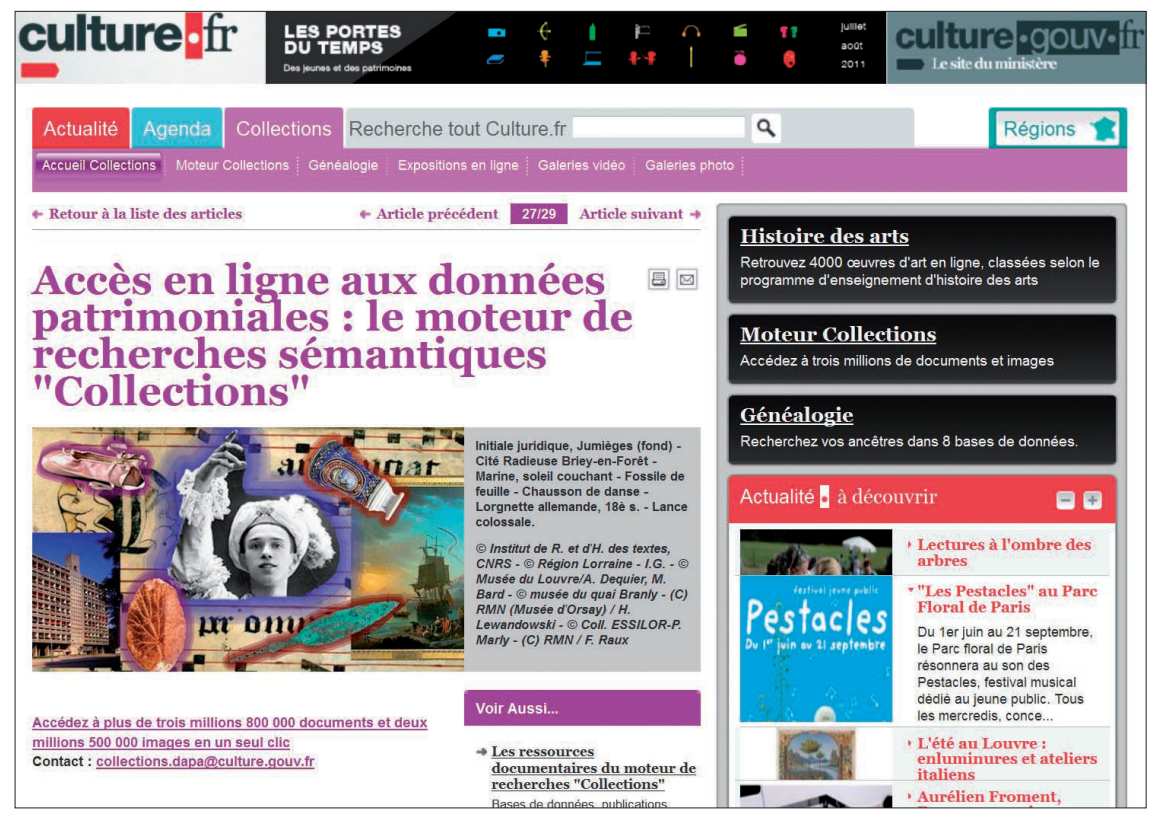

http://www.culture.fr/fr/sections/collections/accueil/presentation_collection bibliotecas para compartir al menos instalaciones, pero frecuentemente también otros servicios, conocidos en la literatura profesional como jointuse libraries, combined libraries o dual use libraries, denominaciones que han sido traducidas al español como bibliotecas integradas o bibliotecas de doble uso o duales ${ }^{1}$.

Este modelo bibliotecario se basa en la alianza entre distintas tipologías de bibliotecas (escolar y pública, pública y universitaria, nacional y universitaria, etc.) que unen sus esfuerzos para atender públicos tradicionalmente considerados diversos, esperando obtener de su fusión sinergias que redundan en la calidad y eficacia de los servicios. Con frecuencia la alianza tuvo su origen en el tamaño limitado de la comunidad a la que servían las bibliotecas implicagencia de la esfera pública-, se pasa en los dos últimos siglos a una tipología más amplia: bibliotecas nacionales, universitarias, parlamentarias, públicas, populares, etc., por más que agrupadas en grandes tipos como los establecidos por la Unesco o la IFLA. Por diversos factores, desde el siglo $\mathrm{XIX}$, pero especialmente en el XX, se han llevado a cabo proyectos cooperativos de muy distintas clases entre diversos tipos de bibliotecas. Con carácter más puntual, en general, no son desconocidas acciones cooperativas entre las bibliotecas y otros tipos de instituciones, entre ellas los archivos y museos, pero en ambos casos cada una de las instituciones participantes ha mantenido su propia identidad.

Algunas tendencias que hemos visto en los últimos decenios en el campo económico, industrial y en las nuevas tecnologías, han impactado de forma considerable en los sectores informativos y culturales. Recordemos brevemente los movimientos producidos en la industria editorial, uno de los proveedores de las bibliotecas. En las décadas de los 80 y los 90 se comienzan a crear grandes grupos editoriales, a partir de dos tipos de concentraciones. En unos casos las fusiones ocurren dentro del mismo sector y son concentraciones horizontales en las que por ejemplo se constituye un gran grupo mediático a partir de la fusión de prensa, TV, radio, etc. EI segundo tipo de fusiones son las concentraciones verticales, que agrupan empresas originalmente de distintos sectores y permiten al grupo resultante de la fusión situar el mismo producto cultural en distintos circuitos culturales: el libro se convierte en un guión de cine, cuya película se exhibe en salas, se vende en DVD, en TV por cable o previo pago, se comercializa su banda musical, etc.

Si nos acercamos al mundo de las bibliotecas y miramos con cierta atención no dejaremos de observar algunas tendencias similares. Esta aproximación es el objeto de este trabajo.

\section{Alianzas horizontales: las bibliotecas integradas}

A lo largo del siglo $\mathrm{XX}$ se han ido desarrollando con éxito desigual proyectos cooperativos entre distintos tipos de das, o al menos una de ellas, y en otros factores tales como las malas comunicaciones y el carácter aislado de una población, especialmente en los sectores donde han tenido un mayor desarrollo, caso de las bibliotecas públicas y escolares. También las limitaciones de recursos económicos y humanos han jugado un papel decisivo en muchas de estas alianzas.

A partir de los años 90 del pasado siglo se produce una evolución de la educación de la población que alcanza tasas de participación en la educación universitaria nunca antes alcanzadas, al tiempo que la formación permanente se considera requisito indispensable para el desarrollo económico, social e individual. En este nuevo nivel formativo de la sociedad las nuevas tecnologías desempeñan un papel central, como muestra la propia expresión "alfabetización informacional". Y es en este contexto donde se explican las cada vez más frecuentes alianzas entre bibliotecas académicas y bibliotecas públicas.

Los inicios de tales bibliotecas pueden ser rastreados en muchos países a partir del siglo XVIII, donde las que hoy son bibliotecas universitarias nacen en España con el expreso papel de atender al público general (desde luego, un público erudito), continúan en el siglo XIX, como en el caso de Suecia donde la orden de 1846 de crear bibliotecas escolares se plasma en muchas ocasiones en el establecimiento de la biblioteca parroquial en la escuela al servicio de ambas comunidades (Hansson, 2006), se encuentra desde principios del siglo XX en USA y desde 1940 en Canadá, aunque han recibido un fuerte impulso desde los años 90 . Su presencia es significativa en algunos países como Estados Unidos, Reino Unido, Canadá, Australia, Suecia y Nueva Zelanda. También en España han surgido ejemplos en los últimos años, el Grupo de Trabajo Sistemas Urbanos de Bibliotecas, creado a partir de la Jornadas de cooperación bibliotecaria 2000, propuso nuevas alianzas entre distintos tipos de bibliotecas urbanas (Jornadas, 2005), y la Ley de la lectura, el libro y las bibliotecas en su artículo 3.3 señala: “...el Gobierno apoyará e incentivará la apertura de las bibliotecas escolares a la comunidad de ciudadanos de su entorno...". Advertir también 
que esta convergencia no es exclusiva de las bibliotecas, sino que otras instituciones culturales parecen caminar en igual sentido: el reciente acuerdo de compartir colecciones y proyectos entre el Macba y la Fundación La Caixa, o la Red de Museos Locales de Catalunya que busca fórmulas de cooperación supramunicipal basadas en la colaboración horizontal, no difieren esencialmente, a no ser en lo que se refiere al espacio común, del modelo de las bibliotecas integradas.

Llegados aquí cabe formularse la pregunta de si emerge un nuevo modelo de bibliotecas, o estamos hablando de modificaciones de un modelo histórico o simplemente de una nueva forma de colaboración. El modelo se diferencia de otros basados en la cooperación

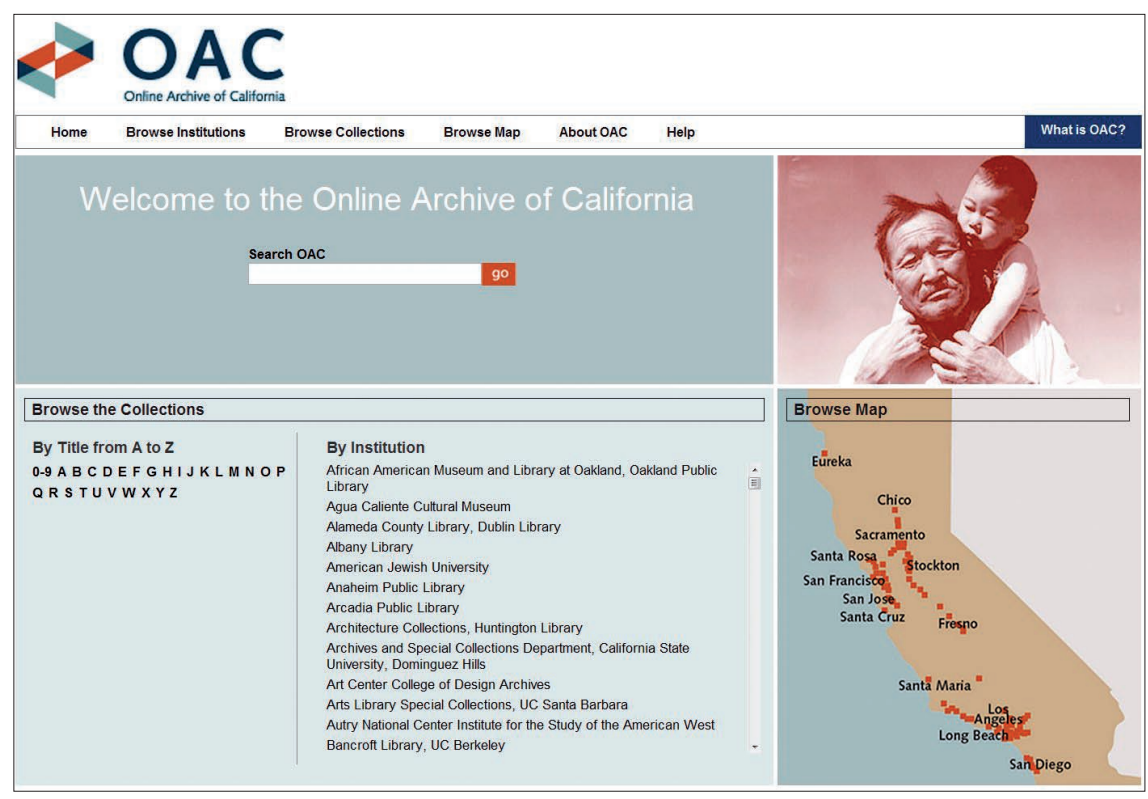

http://www.oac.cdlib.org (escuela-biblioteca pública, redes de bibliotecas, etc.) en la medida en que las alianzas son imprescindibles al nivel de las administraciones que sustentan a las bibliotecas, en que las bibliotecas comparten un espacio común, y todo ello basado en un acuerdo que especifica sus relaciones. En éste se señala para cada participante el papel y responsabilidad, cómo se resolverán los problemas no previstos, sus funciones en la gestión y su aportación a los recursos financieros del nuevo servicio.

Estas alianzas adoptan distintos grados de integración, que van desde la existencia de co-directores que representan a los distintos servicios participantes y asumen la responsabilidad de liderar y organizar las distintas actividades, hasta la configuración de una dirección común e integración de operaciones de los distintos servicios relacionados, pasando por una dirección común que gestiona en exclusiva los recursos económicos y humanos de los distintos servicios, o variantes de algunas de las indicadas. Sin duda, es la penúltima tendencia la que proporciona más rápidamente una nueva identidad a la alianza resultante, pero sospechamos que en cualquiera de los marcos que venimos de señalar se producirá un cambio identitario.

Pero además cabe analizar el modelo en relación con otras alianzas que se observan en el ámbito cultural, ya señaladas, respuesta a la globalización o internacionalización de la economía, en la que las TIC han jugado un papel imprescindible para su desarrollo. No cabe duda de que las bibliotecas integradas pueden ser vistas como alianzas horizontales entre bibliotecas, y quizá debemos preguntarnos si tales alianzas responden a la necesidad de hacer frente a las alianzas del mercado cultural, aglutinado alrededor del formato digital, lo que pensamos caracteriza también a otras formas cooperativas, tales como los consorcios ${ }^{2}$. Si esto es así, y dado que no se vislumbra el camino hacia formas distintas de organización económica, debemos pensar que existe un largo trayecto para el desarrollo de bibliotecas integradas.

Las nuevas bibliotecas integradas se nos muestran así como la ruptura de unas fronteras definidas entre los distintos

tipos de bibliotecas, o si se prefiere su especialización en función de los distintos tipos de usuarios, respondiendo mejor a una sociedad con un nivel formativo más alto, a las posibilidades de empaquetar de formas diversas la misma información digital y a un mercado monopolizado por las fusiones de la industria cultural.

\section{Alianzas verticales y convergencia}

Si las fronteras entre los distintos tipos de bibliotecas hace ya años que comenzaron a diluirse, también las bibliotecas han comenzado en los últimos tiempos a desarrollar alianzas con servicios que tradicionalmente eran disjuntos dentro del sector. El ejemplo del nuevo modelo de biblioteca universitaria denominado learning resources centre en UK, o centro de recursos para el aprendizaje y la investigación (crai) en España, muestra algunas de estas alianzas, que comienzan con las de bibliotecas y servicios informáticos y se expanden a servicios de información y orientación a la comunidad universitaria, de publicaciones, laboratorios de idiomas, servicios de formación del profesorado, etc. En España el modelo ha sido asumido ya por Rebiun (Red de Bibliotecas Universitarias) (Area, 2005).

En Europa el Reino Unido lidera este tipo de fusiones, seguido de Finlandia y Alemania, y en España emerge sobre todo en Catalunya.

En estas alianzas son las bibliotecas y los centros tecnológicos universitarios los participantes más típicos, y el servicio siempre combina un espacio físico, habitualmente la biblioteca, una variedad de colecciones informativas físicas, electrónicas y virtuales, servicios de tecnologías avanzadas y formación amplia en las áreas implicadas (aprendizaje, habilidades de escritura, usos tecnológicos, investigación). Se le reconoce como su mayor éxito la residencia de todos estos servicios en una única localización dentro del campus.

El nuevo modelo de servicio comienza a hacerse sitio. En los campus de los EUA aparecen las denominaciones information commons y learning commons, que destacan su papel 


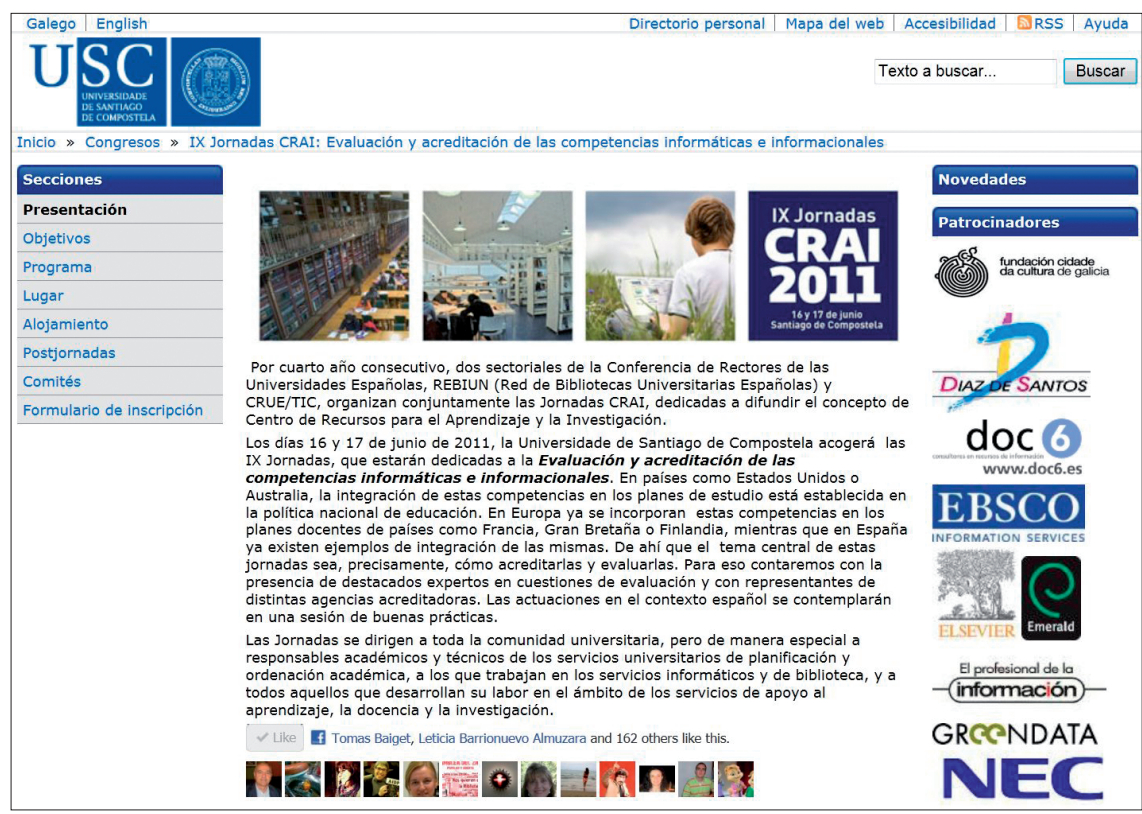

Web de las IX Jornadas crai organizadas por la Universidade de Santiago de Compostela http://www.usc.es/es/congresos/crai/index.html

comunitario. Ambos términos son usados indistintamente y también como sinónimos de learning resources center, considerándose que los dos últimos son términos más genéricos, con menor presencia de los aspectos tecnológicos. Estos servicios van generalmente unidos a las bibliotecas, donde sustituyen los servicios de referencia (Lippincott, 2006), aunque para algunos autores son una reformulación de la propia biblioteca (Remy, 2004). Sus diferencias con las bibliotecas son fundamentalmente de escala en el uso de los recursos tecnológicos: más hardware y sobre todo un uso intensivo de software para el acceso a la información y la producción de información, preferentemente en grupo, respondiendo así a un modelo de enseñanza que se basa más en el aprendizaje del alumno que en la lección magistral del profesor. Naturalmente el modelo se basa en la interacción de dos tipos de personal hasta ahora tradicionalmente distinto, que trabaja conjuntamente o de forma separada para proporcionar los servicios, pero siempre compartiendo el espacio hasta el punto de que con frecuencia se definen como one stop-shopping (lugar donde se encuentra todo lo necesario). En este sentido, al igual que las bibliotecas integradas, el modelo entraría dentro de la nueva estrategia que ha sido denominada "la biblioteca como espacio".

Pero hay otro sector emergente en las alianzas verticales de las bibliotecas. El ya señalado trío de opuestos manuscrito / impreso / objeto, si ya carecía de rigor aplicado a los soportes y formatos tradicionales, parece difuminarse más con la introducción de los formatos audiovisuales en sus colecciones (archivos orales, videotecas, videoarte...), y ahora en el común soporte digital. Por otra parte, las componentes informativas, educativas, y educativo-recreativas (endutainment) que las bibliotecas, archivos y museos han adquirido a lo largo del siglo XX los han acercado en las políticas de información, gestión, aplicaciones tecnológicas, y en la conservación y puesta en servicio del patrimonio cultural. En la actualidad este acercamiento es visible en un gran número de acciones cooperativas con vistas a la formación permanente, pero también al desarrollo económico, en países como Noruega, Gran Bretaña, Estados Unidos y Canadá, tanto en colaboraciones puntuales como permanentes.

En EUA el Institute of Museum and Library Services (IMLS), en Canadá Library and Archives Canada (LAC), en UK Museums, Libraries \& Archives (MLA) son agencias gubernamentales cuyo nombre deja claramente expuesto el acercamiento entre instituciones, a las cuales lideran, coordinan y financian. Por su parte, los organismos internacionales, como el Consejo de Europa llaman a las autoridades nacionales a la convergencia en el marco de las políticas nacionales e internacionales (Consejo de Europa, 2000).

En el campo normativo en España también se dan pasos hacia el acercamiento de las tres instituciones, como muestra la Ley $11 / 2007$ de Bibliotecas de Euskadi que señala en su artículo 5 que los servicios bibliotecarios se prestarán mediante una política de información dirigida a la convergencia de las instituciones de los sectores de archivos, bibliotecas y museos. Además no faltan propuestas para una convergencia más profunda con vistas a la creación de un nuevo modelo de instituciones híbridas, o, como se las ha denominado, "infraestructuras transinstitucionales". Siguiendo este nuevo modelo, entre 2004 y 2006 Bibliothèque et Archives nationales du Québec (BAnQ) unificó sus funciones de biblioteca nacional, biblioteca pública y archivos nacionales en una única institución.

Los profundos cambios que las nuevas tecnologías de la información y la comunicación están ocasionando sobre estas instituciones, sobre sus colecciones, la organización de las mismas y su puesta en servicio ofrece un potencial importante para una convergencia, al menos en el campo digital (Hedstrom; King, 2004), señalando de nuevo un espacio único en la Red. Si analizamos las colecciones, en el mundo digital la diferenciación tradicional de soportes ha desaparecido, aunque sin duda el atractivo del objeto físico sea mayor en los museos que en las bibliotecas o los archivos. Mientras que la consulta del documento en red de las bibliotecas y los archivos es suficiente para muchos usuarios, y el espacio físico en las bibliotecas se transforma disminuyendo su valor como lugar de la información y potenciando su función de espacio comunitario (lugar de encuentro, espacio de trabajo en grupo...), la consulta de la pieza museográfica digitalizada no disminuye la atracción del objeto físico, y en esa medida no modifica el museo como espacio.

En relación a los formatos, la Red evoluciona del texto a la imagen en movimiento, de la escritura al vídeo, etc., haciéndose audiovisual, algo ya claramente perceptible en bibliotecas, archivos y museos, como por ejemplo en los podcasts, webcasts, canales de TV de la Library of Congress o de la Tate Gallery, por citar dos instituciones de alcance internacional, pero también en centros locales, como el Archivo de Arganda del Rey, Madrid. 
En cuanto a la recuperación de la información, el sistema de búsqueda en Red, de los buscadores, directorios, metabuscadores, etc., se ha convertido en el paradigma de la búsqueda y recuperación de la información, de "toda" la información, física o virtual, y esta perspectiva a corto plazo va a condicionar el trabajo de las instituciones de la memoria, en algunos países en fase de debate, a veces en franca convergencia. Están siendo llevados adelante esfuerzos para unificar o interrelacionar procedimientos entre los tres tipos de instituciones, y se trabaja sobre el acceso transversal a los contenidos culturales de las tres.

Ejemplos de ello son:

- el portal alemán BAM ${ }^{3}$ (Bibliotheken, Archive or Museen) que permite la búsqueda común a partir de metadatos de catálogos e inventarios de fondos de bibliotecas, archivos y museos y otras instituciones alemanas que poseen bienes culturales, con más de 40 millones de registros. Las búsquedas están enlazadas con las interfaces originales donde se consultan también los documentos digitalizados y cuyo acceso es posible también a partir de la Wikipedia alemana desde puntos de acceso coincidentes;

- Culture.fr que a partir del motor "Collections" interroga simultáneamente las diferentes fuentes documentales del Ministerio de Cultura francés (BnF, Archives Departamentales, Musée du Louvre, bibliotecas municipales...), u

- OAC ${ }^{5}$ (Online Archive of California), donde más de 150 bibliotecas, archivos, colecciones especiales y sociedades de historia constituyeron un espacio web con descripciones detalladas de fuentes primarias (objetos artísticos, manuscritos, fotografías,...), y en muchos casos con acceso a las piezas digitalizadas.

Europeana ${ }^{6}$, la biblioteca digital europea, también junta en sus colecciones archivos, bibliotecas, museos y colecciones audiovisuales.

\section{Conclusiones}

Los modelos vistos hasta aquí pueden tener un largo o un corto recorrido, pero parece claro que emergen nuevos modelos bibliotecarios y que en ellos el concepto de biblioteca que hemos visto desarrollarse en los últimos dos siglos tiende a tener cada vez más unas fronteras indefinidas ${ }^{7}$, y aplica nuevas políticas de alianzas.

Si bien es cierto que los acuerdos de cooperación son conocidos desde hace tiempo, se hacían generalmente entre bibliotecas del mismo tipo, o bien para fines concretos (adquisiciones, préstamo interbibliotecario...). Pero en una sociedad que está en proceso de redefinición, el nuevo orden digital en que la información y el conocimiento se empaquetan y distribuyen en formas diversas pero que tienen en común ser digitales, obliga a profundas mutaciones en las

\section{CombiLib}

\section{mbined Libmries in the Baltic and Norfic conntries}

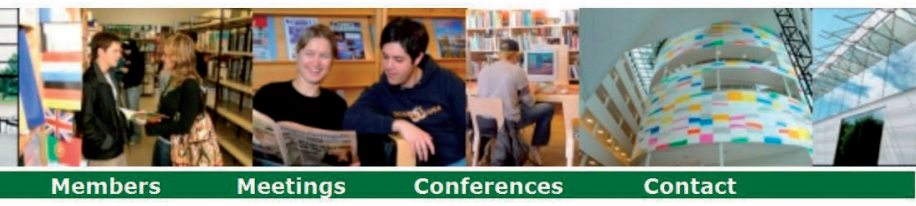

Meeting in Härnösand, 20th-21th October 2011

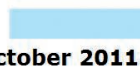

\section{Meeting in Harnosand, 20 th-21th}

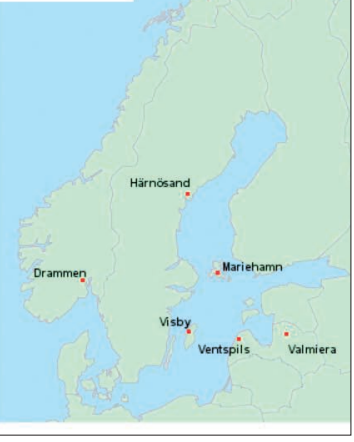

nuevas alianzas de las bibliotecas, algo que previamente se ha plasmado en otros sectores relacionados con ellas, como el de las industrias culturales.

\section{Notas}

1. Para la operatividad y puesta en marcha de bibliotecas integradas entre bibliotecas públicas y bibliotecas de enseñanza nos parece especialmente interesante DasGupta, Kalpana (2009).

2. Las propias bibliotecas integradas han desarrollado entre ellas formas de cooperación tradicionales, tales como la red de bibliotecas nórdicas CombiLib http://www.combilib.net/index.html

\section{Bibliotheken, Archiven, Museen (Alemania) http://www.bam-portal.de}

\section{Collections (Francia) \\ http://www.culture.fr/fr/sections/collections/accueil/presen tation_collection}

\section{Online Archive of California http://www.oac.cdlib.org}

\section{6. http://www.europeana.eu/portal}

7. Sería muy interesante estudiar el impacto de los nuevos modelos sobre el personal. Una aproximación a este estudio puede verse en Sennyey, Pongracz et al. (2009).

\section{Bibliografía}

Area, Manuel (coord.). De las bibliotecas universitarias a los centros de recursos para el aprendizaje y la investigación. Resumen del informe financiado por el Ministerio de Educación y

Ciencia. Rebiun, Documentos de trabajo, Crue (Conferencia de Rectores de Universidades Españolas), 2005, 88 pp. ISBN: 85-932783-4-3 http://www.rebiun.org/doc/z2.pdf 
Bastide, Roger. Anthropologie appliquée. Paris: Stock, 1998. ISBN: 2234049032

Bourdieu, Pierre. Raisons pratiques: sur la théorie de I'action. Paris: Editions du Seuil, 1994. ISBN: 2020231050

Consejo de Europa. Comité de Cultura. Council of Europe/ Eblida guidelines on library legislation and policy in Europe, 2000.

http://www.coe.int/t/dg4/cultureheritage/culture/resour ces/DECS_CULT_POL_book\%282000\%291_EN.pdf

Chapron, Emmanuelle. "Ad utilità pubblica": politique des bibliothèques et pratiques du libre à Florence au XVIII siècle. Genève: Droz, 2009. ISBN: 9782600012355

DasGupta, Kalpana. "Towards an inclusive knowledge society: role of joint use libraries in Indian academic and public library sectors". En: Int/ conf on academic libraries (ICAL 2009). Delhi.

http://crl.du.ac.in/ical09/papers/index_files/ical-80_228_ 484_4_RV.pdf

Foucault, Michel. Las palabras y las cosas: una arqueología de las ciencias humanas. México: Siglo Veintiuno, 1968. ISBN: 9682300177

Gramsci, Antonio. II materialismo storico e la filosofía de Benedetto Croce. 10a ed. Torino: Giulio Einaudi, 1974.

Hansson, Joacim. "Just collaboration or really something else? On joint use libraries and normative institutional change with two examples from Sweden". Library trends, 2006, v. 54, n. 4, pp. 549-568.

http://findarticles.com/p/articles/mi_m1387/is_4_54/ai_ n26915949
Hedstrom, Margaret; King, John-Leslie. "On the LAM: library, archive, and museum collections in the creation and maintenance of knowledge communities". En: Mapping innovation: six depth studies. Organization for Economic Cooperation and Development, 2004.

http://www.oecd.org/dataoecd/59/63/32126054.pdf

"Sistemas urbanos de bibliotecas". En: Jornadas de cooperación bibliotecaria. Grupo de Trabajo de Sistemas Urbanos de Bibliotecas, 2005

http://travesia.mcu.es/portalnb/jspui/bitstream/10421/ 389/1/SistemasUrbanos.pdf

Lippincott, Joan K. "Linking the information commons to learning". En: Oblinger, Diana (ed.). Learning spaces. Educase, 2006. ISBN: 0967285372

http://net.educause.edu/ir/library/pdf/PUB7102g.pdf

Merton, Robert. Social theory and social structure. New York: The Free Press, 1968. ISBN: 9780029211304

Remy, Melanie. Information literacy: the information commons connection, 2004.

http://www.usc.edu/libraries/locations/leavey/new_at leavey/conference/presentations/presentations_9-17/USC_ Remy.pdf

Sennyey, Pongracz; Ross, Lyman; Mills, Caroline. "Exploring the future of academic libraries: A definitional approach". Journal of academic librarianship, 2009, v. 35, n. 3, pp. 252259.

Varela-Orol, Concha. "Librerías y bibliotecas: el nacimiento de las bibliotecas públicas en España". Bulletin hispanique, 2009 , v. 111 , n. 2 , pp. 429-447.

\section{IraLIS es el registro de autoridades de nombres de autor del repositorio E-LIS}

\section{Antes de subir documentos a E-LIS debes iralizarte siguiendo los "Criterios de firma".}

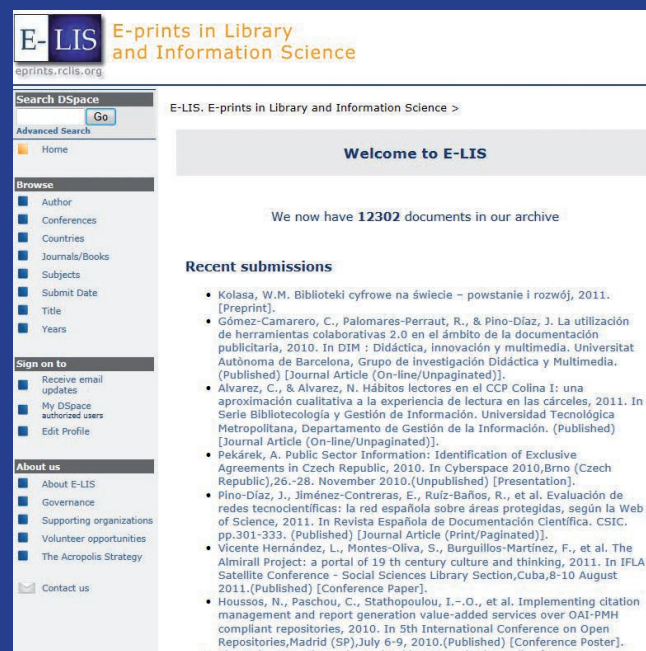

http://eprints.rclis.org

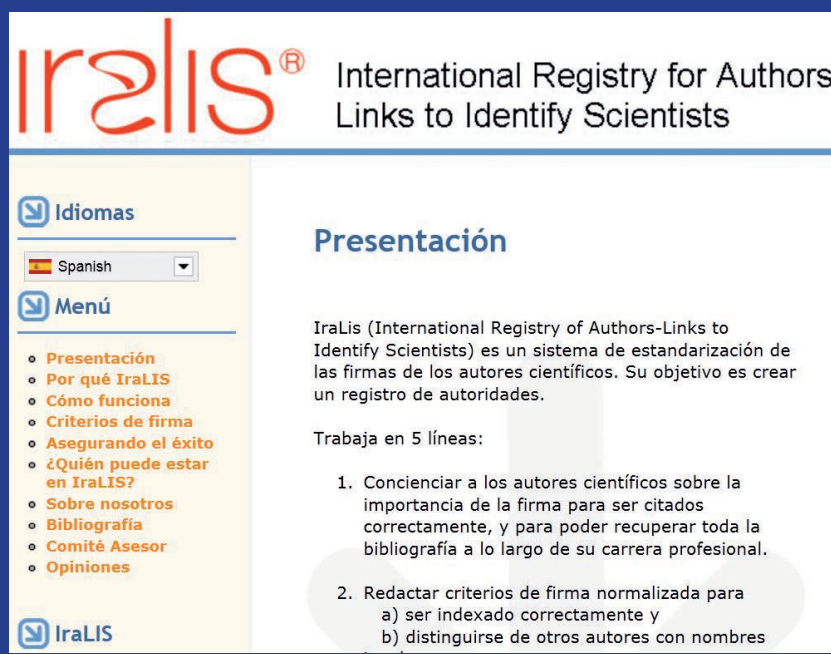

http://www.iralis.org 